\title{
P-75
}

\section{Zerumbone in Combination with Curcumin FOR Probable Treatment in Liver Cancer}

\author{
Nozlena A. Samad ${ }^{1}$, Ahmad Bustamam Abdul ${ }^{1,}$, Heshu Rahman ${ }^{2}$, A. Reena Joys ${ }^{1}$, Rasedee \\ Abdullah $^{2}$, Tengku Azmi Tengku Ibrahim ${ }^{2}$ and S.K. Yeap ${ }^{1}$ \\ ${ }^{1}$ Institute of Bioscience, University Putra Malaysia, 43400 UPM Serdang, Selangor, Malaysia; ${ }^{2}$ Faculty of Veterinary \\ Medicine, Department of Microbiology and Pathology, University Putra Malaysia, 43400 UPM Serdang, Selangor, \\ Malaysia; E-mail:ahmadbstmm@yahoo.com
}

There has been increasing evidence that cancers are dependent on a number of altered molecular pathways, which can develop diverse mechanisms of resistance to therapy with single agents. Therefore, combination regimens may provide the best hope for effective therapies with durable effects. In this current investigation, the anti-cancer effect of zerumbone (ZER), a natural compound from Zingiber zerumbet smith of Zingiberaceae family and curcumin (a yellow pigment from Curcuma longa) were used in combination for probable treatment in liver cancer. Both compounds were investigated in MTT cell proliferative assay as well as flow cytometric analysis (annexinV-FITC, cell cycle and TUNEL assay) on HepG2 cells in 3 ratios of ZER: CUR $(1: 3,3: 1$ and $1: 1)$. The $\mathrm{IC}_{50}$ obtained were $13 \mu \mathrm{g} / \mathrm{mL}, 4 \mu \mathrm{g} / \mathrm{mL}$ and $7 \mu \mathrm{g} / \mathrm{mL}$ respectively and the combinations further showed no toxic effect on normal liver cell lines (WRL68). The current findings revealed that the best combination of ZER: CUR is 3:1. The outcome of this study demonstrates that this combination has the ability to increased efficacy with limited toxicity in liver cancer treatment.

Keywords: Zerumbone, Curcumin, Cell proliferative assay, Flow cytometry analysis, Liver Cancer. 